\title{
Liftings in Finite Graphs and Linkages in Infinite Graphs with Prescribed Edge- Connectivity
}

\author{
Ok, Seongmin; Richter, R. Bruce; Thomassen, Carsten
}

Published in:

Graphs and Combinatorics

Link to article, DOI:

$10.1007 / \mathrm{s} 00373-016-1724-9$

Publication date:

2016

Document Version

Peer reviewed version

Link back to DTU Orbit

Citation (APA):

Ok, S., Richter, R. B., \& Thomassen, C. (2016). Liftings in Finite Graphs and Linkages in Infinite Graphs with Prescribed Edge-Connectivity. Graphs and Combinatorics, 32(6), 2575-2589. https://doi.org/10.1007/s00373016-1724-9

\section{General rights}

Copyright and moral rights for the publications made accessible in the public portal are retained by the authors and/or other copyright owners and it is a condition of accessing publications that users recognise and abide by the legal requirements associated with these rights.

- Users may download and print one copy of any publication from the public portal for the purpose of private study or research.

- You may not further distribute the material or use it for any profit-making activity or commercial gain

- You may freely distribute the URL identifying the publication in the public portal 


\title{
Liftings in finite graphs and linkages in infinite graphs with prescribed edge-connectivity*
}

\author{
Seongmin $\mathrm{Ok}^{\dagger}$, R. Bruce Richter ${ }^{+\ddagger}$, and Carsten Thomassen ${ }^{\dagger \circ}$ \\ LTEX-ed: June 20, 2016
}

\begin{abstract}
Let $G$ be a graph and let $s$ be a vertex of $G$. We consider the structure of the set of all lifts of two edges incident with $s$ that preserve edge-connectivity. Mader proved that two mild hypotheses imply there is at least one pair that lifts, while Frank showed (with the same hypotheses) that there are at least $(\operatorname{deg}(s)-1) / 2$ disjoint pairs that lift. We consider the lifting graph: its vertices are the edges incident with $s$, two being adjacent if they form a liftable pair. We have three main results, the first two with the same hypotheses as for Mader's Theorem.

(i) Let $F$ be a subset of the edges incident with $s$. We show that $F$ is independent in the lifting graph of $G$ if and only if there is a single edge-cut $C$ in $G$ of size at most $r+1$ containing all the edges in $F$, where $r$ is the maximum number of edge-disjoint paths from a vertex (not $s$ ) in one component of $G-C$ to a vertex (not $s$ ) in another component of $G-C$.

(ii) In the $k$-lifting graph, two edges incident with $s$ are adjacent if their lifting leaves the resulting graph with the property that any two vertices different from $s$ are joined by $k$ pairwise edge-disjoint paths. If $\operatorname{both} \operatorname{deg}(s)$ and $k$ are even, then the $k$-lifting graph is a connected complete multipartite graph. In all other cases, there are at most two components. If there are exactly two components, then each component is a complete multipartite graph. If $\operatorname{deg}(s)$ is odd and there are two components, then one component is a single vertex.

(iii) Huck proved that if $k$ is odd and $G$ is $(k+1)$-edge-connected, then $G$ is weakly $k$-linked (that is, for any $k$ pairs $\left\{x_{i}, y_{i}\right\}$, there are $k$ edge-disjoint paths $P_{i}$, with $P_{i}$ joining $x_{i}$ and $y_{i}$ ). We use our results to extend a slight weakening of Huck's theorem to some infinite graphs: if $k$ is odd, every $(k+2)$-edge-connected, locally finite, 1-ended, infinite graph is weakly $k$-linked.

Keywords: edge-connectivity, lifting

AMS Classification (2000): 05C40

seok@dtu.dk, brichter@uwaterloo.ca, and ctho@dtu.dk
\end{abstract}

* Some of this work was done by the second and third authors while at the Institut Mittag-Leffler (Djursholm, Sweden). Some of this work was done during a visit by the first author to the University of Waterloo.

${ }^{\dagger}$ Department of Applied Mathematics and Computer Science, Technical University of Denmark, and ${ }^{+}$Department of Combinatorics \& Optimization, University of Waterloo.

Supported in part by ${ }^{\ddagger} \mathrm{NSERC}$ and ${ }^{\circ}$ ERC Advanced Grant GRACOL. 


\section{Introduction}

For distinct vertices $x$ and $y$ in a graph $G, \lambda_{G}(x, y)$ denotes the maximum number of pairwise edge-disjoint $x y$-paths in $G$. We shall assume that $x$ and $y$ have a target connectivity $\tau_{G}(x, y) \leq \lambda_{G}(x, y)$. In the cases of immediate interest, either $\tau_{G} \equiv \lambda_{G}$ or $\tau_{G}$ is constant, but the target unifies and generalizes both these particular cases.

Let $s$ be a vertex of $G$ and let $s v$ and $s w$ be two edges incident with $s$. The lift of $G$ at $s v$ and $s w$ is the graph $G_{v, w}$ obtained from $G-\{s v, s w\}$ by adding the edge $v w$.

The lift of $G$ at $s v$ and $s w$ is $\tau_{G}$-feasible if, for every pair $x, y$ of distinct vertices in $G-s, \lambda_{G_{v, w}}(x, y) \geq \tau_{G}(x, y)$. We will just say feasible, since $\tau_{G}$ will always be understood.

Let $s$ be a vertex in a graph $G$ that does not have degree 3 and is not incident with an isthmus. (An isthmus is an edge whose deletion from $G$ increases the number of components.) Mader [5] proved (for target $\lambda_{G}$ and therefore for any target) that there is always a feasible lift in $G$ using two edges incident with $s$. Frank [3] extended this to show that there are $\lfloor\operatorname{deg}(s) / 2\rfloor$ pairwise disjoint such feasible pairs.

For any subset $A$ of $V(G)$, we set $\delta_{G}(A)$ to be the set of edges of $G$ having one end in $A$ and one end not in $A$. By Menger's Theorem, the obstruction to $s v$ and $s w$ yielding a feasible lift is that there is a pair $a, b$ of vertices and a set $A$ of vertices so that $a \in A, b, s \notin A$, and $\left|\delta_{G_{v, w}}(A)\right|<\tau_{G}(a, b)$. Since obviously $\left|\delta_{G}(A)\right| \geq \tau_{G}(a, b)$ and $\left|\delta_{G_{v, w}}(A)\right| \geq\left|\delta_{G}(A)\right|-2$, we see that $\left|\delta_{G}(A)\right| \leq\left|\tau_{G}(a, b)\right|+1$. Thus motivates the following important notion.

Let $A$ be a subset of $V(G) \backslash\{s\}$. Then $r(A)$ is defined to be $\max \left\{\tau_{G}(a, b) \mid a \in A, b \notin\right.$ $A \cup\{s\}\}$. Also, $A$ is a dangerous set if $\left|\delta_{G}(A)\right| \leq r(A)+1$. The preceding paragraph readily implies the observation that $s v$ and $s w$ do not have a feasible lift if and only if there is a dangerous set $A$ such that $v, w \in A$.

Henceforth, all considerations are in $G$, so we write $\delta(A)$ instead of $\delta_{G}(A)$.

The first of our three main results is the following. The "if" part of the statement is trivial; the "only if" is proved in the next section.

Theorem 1.1 Let $G$ be a graph and let $s$ be a vertex of $G$ that does not have degree 3 and is not incident with an isthmus. Let $F$ be any set of at least two edges, all incident with s. Then no pair of edges in $F$ yields a feasible lift if and only if there is a dangerous set $A$ so that, for every $s v \in F, v \in A$.

Let $G$ be a graph, let $s$ be a vertex of $G$, and let $\tau$ be the edge-connectivity target function. The lifting graph $L(G, s, \tau)$ has as its vertices the edges of $G$ incident with $s$ and two edges are adjacent in $L(G, s, \tau)$ if they form a $\tau$-feasible pair. If there is a positive 
integer $k$ so that $\tau \equiv k$, then we write $L(G, s, k)$ for $L(G, s, \tau) ; L(G, s, k)$ is the $k$-lifting graph.

Thomassen [8] proved that the $k$-lifting graph of an Eulerian graph has a disconnected complement. This was used to prove a decomposition theorem for infinite graphs that implies, among other things, a conjecture from 1989: every $8 k$-edge-connected infinite graph has a $k$-arc-connected orientation.

Part (1.2.4) of our second main result generalizes Thomassen's Eulerian result to the $k$-lifting graph when $\operatorname{deg}(s)$ and $k$ are both even.

Theorem 1.2 Let $G$ be a graph with a vertex $s$ and let $k$ be a positive integer such that any distinct vertices different from $s$ are joined by $k$ pairwise edge-disjoint paths. If $s$ is not incident with an isthmus and $\operatorname{deg}(s) \geq 4$, then:

(1.2.1) the k-lifting graph $L(G, s, k)$ has at most two components;

(1.2.2) if $\operatorname{deg}(s)$ is odd and $L(G, s, k)$ has two components, then one has only one vertex and the other component is complete multipartite;

(1.2.3) if $\operatorname{deg}(s)$ is even and $L(G, s, k)$ has two components, then each component is complete multipartite with an even number of vertices; and

(1.2.4) if $\operatorname{deg}(s)$ and $k$ are both even, then $L(G, s, k)$ is a connected, complete multipartite graph (in particular, it has a disconnected complement).

If either $L(G, s, k)$ is not connected or both $\operatorname{deg}(s)$ and $k$ are even, then any component of $L(G, s, k)$ with at least 4 vertices is not a star $K_{1, r}$.

A graph $G$ is weakly $k$-linked if, for any sequences $x_{1}, x_{2}, \ldots, x_{k}$ and $y_{1}, y_{2}, \ldots, y_{k}$ of (not necessarily distinct) vertices of $G$, there are $k$ edge-disjoint paths $P_{1}, P_{2}, \ldots, P_{k}$ such that $P_{i}$ has ends $x_{i}$ and $y_{i}$. By choosing all the $x_{i}$ to be the same vertex and all the $y_{i}$ to be the same vertex, we see that any weakly $k$-linked graph is $k$-edge-connected. Thomassen [7] conjectured that, when $k$ is odd, the converse holds. Okamura [6] obtained the first significant result about this conjecture (roughly: if $G$ is $\frac{4}{3} k$-edge-connected, then $G$ is weakly $k$-linked). Then Huck [4] proved that, if $k$ is odd and $G$ is $(k+1)$-edge-connected, then $G$ is weakly $k$-linked.

We use Huck's Theorem and Theorem 1.2 (1.2.4) to prove the following. Recall that an infinite graph $G$ is locally finite if, for every vertex $v$ of $G, \operatorname{deg}(v)$ is finite. Also, a graph $G$ is 1-ended if, for every finite set $S$ of vertices, $G-S$ has at most one infinite component. 
Theorem 1.3 Let $k$ be an odd positive integer. If $G$ is a $(k+2)$-edge-connected, 1-ended, locally finite graph, then $G$ is weakly k-linked.

We remark that we can prove that the hypothesis of Theorem 1.3 implies that any $(k+2)$-edge-connected, infinite, locally finite graph with only finitely many ends is weakly $k$-linked. There are some technicalities that are not germane to the application of Huck's Theorem and Theorem 1.2. We believe the following much stronger statement is true and so choose not to include this intermediate result.

Conjecture 1.4 Let $k$ be an odd positive integer. If $G$ is a $(k+2)$-edge-connected (infinite) graph, then $G$ is weakly k-linked.

\section{Characterizing independent sets in the lifting graph}

Our goal in this section is to prove Theorem 1.1. It is evident that, if there is a dangerous set $A$ such that, for every $s v \in F, v \in A$, then no two edges in $F$ give a feasible lift. It was the converse that attracted us.

Chan et al [2] give a very closely related argument, presented very efficiently. Our theorem is used significantly in the next section, so we include our slightly modified version of their proof.

For the proof, it will be helpful to set $\sigma(A)=|\delta(A)|-r(A)$ and $\delta(A, B)$ as the set of edges with one end in $A$ and other end in $B$. We note that $A$ is dangerous if and only if $\sigma(A) \leq 1$. The following observation is due to Frank.

Lemma 2.1 [3, Prop. 2.3] Let $s$ be a vertex in a graph $G$ and let $A$ and $B$ be subsets of $V(G) \backslash\{s\}$. Then either

$$
\begin{aligned}
& \text { (2.1.1) } \sigma(A \cup B)+\sigma(A \cap B)+2|\delta(A \backslash B, B \backslash A)| \leq \sigma(A)+\sigma(B) \text { or } \\
& \text { (2.1.2) } \sigma(A \backslash B)+\sigma(B \backslash A)+2|\delta(A \cap B, V(G) \backslash(A \cup B))| \leq \sigma(A)+\sigma(B) \text {. }
\end{aligned}
$$

The key lemma for our proof is the following variant of [2, Lemma 2.7]. The proof requires only very minor modifications from that in [2].

Lemma 2.2 Let $G$ be a graph and s a vertex of $G$. Suppose sa, sb, and sc are three edges incident with $s$ so that none of the lifts of $\{s a, s b\},\{s a, s c\}$, and $\{s b, s c\}$ is $\tau$-feasible. For $\{x, y, z\}=\{a, b, c\}$, let $D_{x}$ be a dangerous set containing $y$ and $z$. Then either $s$ has degree 3, or $s$ is incident with an isthmus, or there is a dangerous subset of $D_{a} \cup D_{b} \cup D_{c}$ containing all three of $a, b$, and $c$ and at least one of $D_{a}, D_{b}$, and $D_{c}$. 
Proof. If any two of $a, b, c$ are the same, then the result is trivial, so we assume $a, b$, and $c$ are all distinct. We consider two cases.

Case 1: For at least one of the pairs $(A, B)$ from $\left(D_{a}, D_{b}\right),\left(D_{a}, D_{c}\right)$, or $\left(D_{b}, D_{c}\right)$, (2.1.1) holds in Lemma 2.1.

We may choose the labelling of $a, b$, and $c$, so that

$$
\sigma\left(D_{a} \cup D_{b}\right)+\sigma\left(D_{a} \cap D_{b}\right)+2\left|\delta\left(D_{a} \backslash D_{b}, D_{b} \backslash D_{a}\right)\right| \leq \sigma\left(D_{a}\right)+\sigma\left(D_{b}\right) .
$$

As each term on the right side is at most 1, the left-hand side is at most 2. If $D_{a} \cup D_{b}$ is dangerous, then we are done, so we may assume $\sigma\left(D_{a} \cup D_{b}\right) \geq 2$. Therefore, the right-hand side is exactly $2, \sigma\left(D_{a} \cup D_{b}\right)=2, \sigma\left(D_{a} \cap D_{b}\right)=0$, and $\left|\delta\left(D_{a} \backslash D_{b}, D_{b} \backslash D_{a}\right)\right|=0$.

Suppose Lemma 2.1 (2.1.1) holds for $A=D_{a} \cap D_{b}$ and $B=D_{c}$; that is,

$$
\begin{gathered}
\sigma\left(\left(D_{a} \cap D_{b}\right) \cup D_{c}\right)+\sigma\left(\left(D_{a} \cap D_{b}\right) \cap D_{c}\right)+2 \mid \\
\delta\left(\left(D_{a} \cap D_{b}\right) \backslash D_{c}, D_{c} \backslash\left(D_{a} \cap D_{b}\right)\right) \mid \\
\leq \sigma\left(D_{a} \cap D_{b}\right)+\sigma\left(D_{c}\right) .
\end{gathered}
$$

Since $\sigma\left(D_{a} \cap D_{b}\right)=0$, the right side is at most 1 and, therefore, $\left(D_{a} \cap D_{b}\right) \cup D_{c}$ is dangerous, and we are done. Therefore, we may assume Lemma 2.1 (2.1.2) applies to $A=D_{a} \cap D_{b}$ and $B=D_{c}$. In particular, $\sigma\left(D_{c} \backslash\left(D_{a} \cap D_{b}\right)\right) \leq \sigma\left(D_{a} \cap D_{b}\right)+\sigma\left(D_{c}\right)$, showing $D_{c} \backslash\left(D_{a} \cap D_{b}\right)$ is dangerous. (It is evidently not empty, as it contains $a$ and $b$.)

Set $D_{c}^{\prime}=D_{c} \backslash\left(D_{a} \cap D_{b}\right)$. The edges $s a$ and $s b$ show that $\mid \delta\left(\left(D_{a} \cup D_{b}\right) \cap D_{c}^{\prime}, V(G) \backslash\right.$ $\left.\left(D_{a} \cup D_{b} \cup D_{c}^{\prime}\right)\right) \mid \geq 2$. On the other hand, the labelling for this case shows $\sigma\left(D_{a} \cup D_{b}\right) \leq$ $\sigma\left(D_{a}\right)+\sigma\left(D_{b}\right) \leq 2$ and the preceding paragraph shows $\sigma\left(D_{c}^{\prime}\right) \leq 1$. Thus,

$$
2\left|\delta\left(\left(D_{a} \cup D_{b}\right) \cap D_{c}^{\prime}, V(G) \backslash\left(D_{a} \cup D_{b} \cup D_{c}^{\prime}\right)\right)\right| \geq 4>3 \geq \sigma\left(D_{a} \cup D_{b}\right)+\sigma\left(D_{c}^{\prime}\right) .
$$

Consequently, Lemma 2.1 implies

$\sigma\left(\left(D_{a} \cup D_{b}\right) \cup D_{c}^{\prime}\right)+\sigma\left(\left(D_{a} \cup D_{b}\right) \cap D_{c}^{\prime}\right)+2\left|\delta\left(\left(D_{a} \cup D_{b}\right) \backslash D_{c}^{\prime}, D_{c}^{\prime} \backslash\left(D_{a} \cup D_{b}\right)\right)\right| \leq \sigma\left(D_{a} \cup D_{b}\right)+\sigma\left(D_{c}^{\prime}\right)$.

If $\left(D_{a} \cup D_{b}\right) \cup D_{c}^{\prime}$ is dangerous, then we are done, so we may assume $\sigma\left(\left(D_{a} \cup D_{b}\right) \cup D_{c}^{\prime}\right) \geq$ 2. As $\sigma\left(D_{a} \cup D_{b}\right)=2$ and $\sigma\left(D_{c}^{\prime}\right) \leq 1$, we conclude that $\sigma\left(\left(D_{a} \cup D_{b}\right) \cap D_{c}^{\prime}\right) \leq 1$ and $\left|\delta\left(\left(D_{a} \cup D_{b}\right) \backslash D_{c}^{\prime}, D_{c}^{\prime} \backslash\left(D_{a} \cup D_{b}\right)\right)\right|=0$. The inequality shows $\left(D_{a} \cup D_{b}\right) \cap D_{c}^{\prime}$ is dangerous, while $\left|\delta\left(D_{a} \backslash D_{b}, D_{b} \backslash D_{a}\right)\right|=0$ implies $\left|\delta\left(\left(D_{a} \cap D_{c}^{\prime}\right) \backslash\left(D_{b} \cap D_{c}^{\prime}\right),\left(D_{b} \cap D_{c}^{\prime}\right) \backslash\left(D_{a} \cap D_{c}^{\prime}\right)\right)\right|=0$.

We claim that either $s a$ or $s b$ is an isthmus of $G$. We have just seen that $\left(D_{a} \cup D_{b}\right) \cap D_{c}^{\prime}$ is dangerous, so,

$$
\begin{aligned}
1 & \geq \sigma\left(\left(D_{a} \cup D_{b}\right) \cap D_{c}^{\prime}\right) \\
& =\left|\delta\left(\left(D_{a} \cup D_{b}\right) \cap D_{c}^{\prime}\right)\right|-r\left(\left(D_{a} \cup D_{b}\right) \cap D_{c}^{\prime}\right) \mid \\
& \geq\left|\delta\left(D_{a} \cap D_{c}^{\prime}\right)\right|+\left|\delta\left(D_{b} \cap D_{c}^{\prime}\right)\right|-\max \left\{r\left(D_{a} \cap D_{c}^{\prime}\right), r\left(D_{b} \cap D_{c}^{\prime}\right)\right\} \\
& \geq \min \left\{\left|\delta\left(D_{a} \cap D_{c}^{\prime}\right)\right|,\left|\delta\left(D_{b} \cap D_{c}^{\prime}\right)\right|\right\} .
\end{aligned}
$$


Therefore, either $\left|\delta\left(D_{a} \cap D_{c}^{\prime}\right)\right| \leq 1$ or $\left|\delta\left(D_{b} \cap D_{c}^{\prime}\right)\right| \leq 1$. We may choose the labelling of $a$ and $b$ so that the former holds. Since $b \in D_{a} \cap D_{c}^{\prime}$, sb shows $\left|\delta\left(D_{a} \cap D_{c}^{\prime}\right)\right| \geq 1$, so we have $\left|\delta\left(D_{a} \cap D_{c}^{\prime}\right)\right|=1$. Therefore, $s b$ is an isthmus, completing the proof in Case 1.

Case 2: For every one of the pairs $\left(D_{a}, D_{b}\right),\left(D_{a}, D_{c}\right)$, and $\left(D_{b}, D_{c}\right)$, (2.1.2) holds in Lemma 2.1.

The assumption of the case implies that, for example,

$$
\sigma\left(D_{a} \backslash D_{b}\right)+\sigma\left(D_{b} \backslash D_{a}\right)+2\left|\delta\left(D_{a} \cap D_{b}, V(G) \backslash\left(D_{a} \cup D_{b}\right)\right)\right| \leq \sigma\left(D_{a}\right)+\sigma\left(D_{b}\right) \leq 2 .
$$

Since $c \in D_{a} \cap D_{b}$ and $s \in V(G) \backslash\left(D_{a} \cup D_{b}\right),\left|\delta\left(D_{a} \cap D_{b}, V(G) \backslash\left(D_{a} \cup D_{b}\right)\right)\right| \geq 1$. We conclude that $\left|\delta\left(D_{a} \cap D_{b}, V(G) \backslash\left(D_{a} \cup D_{b}\right)\right)\right|=1, \sigma\left(D_{a} \backslash D_{b}\right)=0$, and $\sigma\left(D_{b} \backslash D_{a}\right)=0$.

As in the preceding paragraph, since $b \in\left(D_{a} \backslash D_{b}\right) \cap D_{c}$, we see that $\mid \delta\left(\left(D_{a} \backslash D_{b}\right) \cap\right.$ $\left.D_{c}, V(G) \backslash\left(\left(D_{a} \backslash D_{b}\right) \cup D_{c}\right)\right) \mid \geq 1$. Also, $\sigma\left(D_{a} \backslash D_{b}\right)=0$ and $\sigma\left(D_{c}\right) \leq 1$. Thus, Lemma 2.1 (2.1.2) does not hold for $A=D_{a} \backslash D_{b}$ and $B=D_{c}$. Therefore (2.1.1) holds in Lemma 2.1; in particular, $\sigma\left(\left(D_{a} \backslash D_{b}\right) \cup D_{c}\right) \leq \sigma\left(D_{a} \backslash D_{b}\right)+\sigma\left(D_{c}\right) \leq 1$. That is, $\left(D_{a} \backslash D_{b}\right) \cup D_{c}$ is dangerous. Since this does not contain $c$, we could set $D_{c}^{\prime}=\left(D_{a} \backslash D_{b}\right) \cup D_{c}$ and conduct this argument over again. When we do this, $D_{a} \backslash D_{b} \subseteq D_{c}^{\prime}$, so we may assume this happens in the first place. That is, we may assume $D_{a} \backslash D_{b} \subseteq D_{c}$; likewise, we may assume $D_{c} \backslash D_{a} \subseteq D_{b}$, and $D_{b} \backslash D_{c} \subseteq D_{a}$.

We still have $\left|\delta\left(D_{a} \cap D_{b}, V(G) \backslash\left(D_{a} \cup D_{b}\right)\right)\right|=1$. Likewise both $\mid \delta\left(D_{a} \cap D_{c}, V(G) \backslash\right.$ $\left.\left(D_{a} \cup D_{c}\right)\right) \mid=1$ and $\left|\delta\left(D_{b} \cap D_{c}, V(G) \backslash\left(D_{b} \cup D_{c}\right)\right)\right|=1$ hold. In particular, we know there is only one edge from $s$ to each of $a, b$, and $c$. Also, it follows that $\mid \delta\left(D_{a} \cup D_{b} \cup\right.$ $\left.D_{c}, V(G) \backslash\left(\{s\} \cup D_{a} \cup D_{b} \cup D_{c}\right)\right) \mid=0$.

If $s$ is not incident with an isthmus, then, for every component $K$ of $G-s,|\delta(V(K))| \geq$ 2. Since $\left|\delta\left(D_{a} \cup D_{b} \cup D_{c}\right)\right|=3$ and all edges in $\delta\left(D_{a} \cup D_{b} \cup D_{c}\right)$ are also incident with $s$, we conclude that $G\left[D_{a} \cup D_{b} \cup D_{c}\right]$ is connected and is a component of $G-s$. Therefore, there are two edge-disjoint as-paths in $G\left[\{s\} \cup D_{a} \cup D_{b} \cup D_{c}\right]$.

If the degree of $s$ is not 3 , then we conclude that $G-s$ has at least two components. If $K$ is a component of $G-s$ other than $G\left[D_{a} \cup D_{b} \cup D_{c}\right]$ and $s$ is not incident with an isthmus, then, for any neighbour $t$ of $s$ in $K$, there are two edge-disjoint $t s$-paths in $G[\{s\} \cup V(K)]$. It follows that there are two edge-disjoint at-paths in $G$, showing that $r\left(D_{a} \cup D_{b} \cup D_{c}\right) \geq 2$.

Since $\left|\delta\left(D_{a} \cup D_{b} \cup D_{c}\right)\right|=3$, we conclude that $\sigma\left(D_{a} \cup D_{b} \cup D_{c}\right) \leq 1$. Thus, $D_{a} \cup D_{b} \cup D_{c}$ is dangerous, as required.

The proof of Theorem 1.1 is now quite simple. 
Proof of Theorem 1.1. We proceed by induction on $|F|$, with the cases $|F|=2$ and 3 being, respectively, trivial and an immediate consequence of Lemma 2.2. So assume $|F| \geq 4$, with $F=\left\{s u_{1}, s u_{2}, \ldots, s u_{k}\right\}$. By induction, there are dangerous sets $A_{k-1}$ and $A_{k}$ containing, respectively, all of $F \backslash\left\{s u_{k-1}\right\}$ and $F \backslash\left\{s u_{k}\right\}$. If either $u_{k-1} \in A_{k-1}$ or $u_{k} \in A_{k}$, then we are done, so we may assume neither of these containments occurs.

Because $s u_{k-1}$ and $s u_{k}$ do not make a feasible lift, there is a dangerous set $A$ containing both $u_{k-1}$ and $u_{k}$; among all such dangerous sets, we choose $A$ to be maximal. If, for every $i \in\{1,2, \ldots, k-2\}, u_{i} \in A$, then we are done. Otherwise, there is some $i \in\{1,2, \ldots, k-2\}$ such that $u_{i} \notin A$.

We apply Lemma 2.2 to the pairs $\left\{u_{i}, u_{k-1}\right\},\left\{u_{i}, u_{k}\right\}$, and $\left\{u_{k-1}, u_{k}\right\}$ and the sets $A$, $A_{k-1}$, and $A_{k}$. We conclude that there is a dangerous set $A^{*}$ containing all of $u_{i}, u_{k-1}$, and $u_{k}$ and also containing one of $A, A_{k-1}$, and $A_{k}$.

If $A \subseteq A^{*}$, then, since $u_{i} \in A^{*} \backslash A$, we contradict the maximality of $A$. Therefore, either $A_{k-1}$ or $A_{k}$ is contained in $A^{*}$, from which we conclude that every $u_{j}$ is in $A^{*}$, as required.

\section{Connection in the lifting graph}

In this section, we prove Theorem 1.2 dealing with the structure of the $k$-lifting graph $L(G, s, k)$.

The proofs are inductive and the base cases $\operatorname{deg}(s)=4$ or 5 require some effort. There is one special argument needed for $\operatorname{deg}(s)=6$ when $k$ is odd. The inductive arguments are based on the following simple observation and its contrapositive.

Observation 3.1 If, after lifting the feasible pair $\left\{e_{1}, e_{2}\right\}$, the pair $\left\{e_{3}, e_{4}\right\}$ is feasible, then $\left\{e_{3}, e_{4}\right\}$ is feasible in the original graph.

\subsection{Some general arguments}

In this subsection, we give a few elementary general arguments used later for describing the lifting graph. The first arguments are based on standard methods for "crossing cuts". Let $A_{1}$ and $A_{2}$ be two subsets of $V(G)$. It is an easy exercise to verify that, where $\bar{A}=V(G) \backslash A$,

$$
\begin{aligned}
2\left[\left|\delta\left(A_{1}\right)\right|+\left|\delta\left(A_{2}\right)\right|-\right. & \left.\left(\left|\delta\left(A_{1} \cap A_{2}, \overline{A_{1} \cup A_{2}}\right)\right|+\left|\delta\left(A_{2} \backslash A_{1}, A_{1} \backslash A_{2}\right)\right|\right)\right]= \\
& \left|\delta\left(A_{1} \cap A_{2}\right)\right|+\left|\delta\left(A_{2} \backslash A_{1}\right)\right|+\left|\delta\left(A_{1} \backslash A_{2}\right)\right|+\left|\delta\left(\overline{A_{1} \cup A_{2}}\right)\right| .
\end{aligned}
$$


A typical application will be when all four sets $A_{1} \cap A_{2}, A_{2} \backslash A_{1}, A_{1} \backslash A_{2}$, and $\overline{A_{1} \cup A_{2}}$ are non-empty and $G$ is $k$-edge-connected. In that case, the right-hand side is at least $4 k$. If, for example, both $\delta\left(A_{1}\right)$ and $\delta\left(A_{2}\right)$ have size $k$, we deduce that $\delta\left(A_{1} \cap A_{2}, \overline{A_{1} \cup A_{2}}\right)$ and $\delta\left(A_{2} \backslash A_{1}, A_{1} \backslash A_{2}\right)$ are both empty. Furthermore, it is a routine exercise to verify that this extreme case can only occur with $k$ even.

We will apply a slightly more sophisticated consequence of Equation 3.1.

Lemma 3.2 Let $k$ be a natural number, and let $G$ be a graph with a vertex s such that any two vertices in $G-s$ are joined by $k$ pairwise edge-disjoint paths in $G$. For $i=1,2$, let $F_{i}$ be an independent set in $L(G, s, k)$ of size $r_{i}$ and suppose there is a dangerous set $A_{i}$ so that $F_{i}=\delta(\{s\}) \cap \delta_{G}\left(A_{i}\right)$. Set $\alpha=\left|F_{1} \cap F_{2}\right|$. If $\alpha>0, r_{1}>\alpha, r_{2}>\alpha$, and $\overline{A_{1} \cup A_{2} \cup\{s\}} \neq \varnothing$, then $r_{1}+r_{2} \leq\lfloor\operatorname{deg}(s) / 2\rfloor+2$.

Proof. Observe that: $\left|\delta_{G-s}\left(A_{1}\right)\right| \leq k+1-r_{1} ;\left|\delta_{G-s}\left(A_{2}\right)\right| \leq k+1-r_{2} ; \mid \delta_{G-s}\left(A_{1} \cap\right.$ $\left.A_{2}\right)|\geq k-\alpha ;| \delta_{G-s}\left(A_{2} \backslash A_{1}\right)\left|\geq k-\left(r_{2}-\alpha\right) ;\right| \delta_{G-s}\left(A_{1} \backslash A_{2}\right) \mid \geq k-\left(r_{1}-\alpha\right)$; and $\left|\delta_{G-s}\left(V(G-s) \backslash\left(A_{1} \cup A_{2}\right)\right)\right| \geq k-\left(\operatorname{deg}(s)-\left(r_{1}+r_{2}-\alpha\right)\right)$.

From Equation 3.1, we deduce that $2\left(k+1-r_{1}+k+1-r_{2}\right) \geq(k-\alpha)+\left(k-\left(r_{2}-\alpha\right)\right)+\left(k-\left(r_{1}-\alpha\right)\right)+\left(k-\left(\operatorname{deg}(s)-\left(r_{1}+r_{2}-\alpha\right)\right)\right)$. Rearranging, we see that $\operatorname{deg}(s)+4 \geq 2\left(r_{1}+r_{2}\right)$. Since every term except possibly $\operatorname{deg}(s)$ is even, $\lfloor\operatorname{deg}(s) / 2\rfloor+2 \geq r_{1}+r_{2}$, as required.

Our final preliminary result gives our first glimpse of some structure in $L(G, s, k)$.

Lemma 3.3 Let $k$ be a natural number, and let $G$ be a graph with a vertex $s$ such that any two vertices in $G-s$ are joined by $k$ pairwise edge-disjoint paths in $G$. If $\operatorname{deg}(s)$ is at least 4, then:

(3.3.1) every independent set in $L(G, s, k)$ has size at most $\left\lceil\frac{1}{2} \operatorname{deg}(s)\right\rceil$; and

(3.3.2) if $\operatorname{deg}(s)$ is even and at least 6, then any two distinct independent sets in $L(G, s, k)$ of size $\frac{1}{2} \operatorname{deg}(s)$ are disjoint.

Proof. By Theorem 1.1, an independent set $F$ corresponds to a dangerous set $A$ containing all the non- $s$ ends of the edges in $F$, so $|\delta(A)| \leq k+1$. If $|\delta(\{s\}) \backslash F|<|F|-1$, then $\delta(A \cup\{s\})$ has size at most $k-1$, a contradiction. Thus, $|\delta(\{s\}) \backslash F| \geq|F|-1$, as required for (3.3.1).

Suppose $F_{1}$ and $F_{2}$ are non-disjoint independent sets of size $\frac{1}{2} \operatorname{deg}(s)$, with corresponding dangerous sets $A_{1}$ and $A_{2}$. At most $\operatorname{deg}(s)-1$ of the edges of $\delta(\{s\})$ have one end in 
$A_{1} \cup A_{2}$, so $\overline{A_{1} \cup A_{2} \cup\{s\}} \neq \varnothing$. Also, each of $A_{1} \cap A_{2}, A_{2} \backslash A_{1}$, and $A_{1} \backslash A_{2}$ has an end of an edge in $F_{1} \cup F_{2}$. Since, for $i=1,2$, Lemma 3.3 (3.3.1) implies $F_{i}=\delta(\{s\}) \cap \delta\left(A_{i}\right)$, the hypotheses of Lemma 3.2 are satisfied. However, $r_{1}=\frac{1}{2} \operatorname{deg}(s)=r_{2}$, showing the conclusion of Lemma 3.2 fails, a contradiction that proves (3.3.2).

\section{$3.2 \operatorname{deg}(s)=4$}

In this subsection, we treat the case $\operatorname{deg}(s)=4$. Let $e_{1}, e_{2}, e_{3}, e_{4}$ be the four edges incident with $s$. It is a triviality that if some pair, say $e_{1}, e_{2}$ is feasible, then so is the complementary pair $e_{3}, e_{4}$. It follows that $L(G, s, k)$ is a union of perfect matchings; Mader's Theorem already shows there is at least one such matching in $L(G, s, k)$. Since it has only four vertices, it can only be one of: a perfect matching; a 4-cycle $C_{4}$; and $K_{4}$. These are all realizable. However, when $k$ is even, the perfect matching is not achievable, as we show next.

Proposition 3.4 Let $k$ be a natural number, and let $G$ be a graph with a vertex such that any two vertices in $G-s$ are joined by $k$ pairwise edge-disjoint paths in $G$. If $\operatorname{deg}(s)=4$, then $L(G, s, k)$ is one of: a perfect matching; $C_{4}$; and $K_{4}$. If $k$ is even, then $L(G, s, k)$ is not a perfect matching.

Proof. We only prove the second assertion. Suppose both pairs $e_{1}, e_{2}$ and $e_{1}, e_{3}$ are not feasible. Then there are dangerous sets $A_{2}$ and $A_{3}$ so that the non- $s$ ends of $e_{1}, e_{2}$ are in $A_{2}$ and the non-s ends of $e_{1}, e_{3}$ are in $A_{3}$.

By definition, $\left|\delta_{G}\left(A_{2}\right)\right| \leq k+1$, while the hypothesis implies $\left|\delta_{G}\left(A_{2} \cup\{s\}\right)\right| \geq k$. Therefore, $e_{3}$ and $e_{4}$ have their non- $s$ ends in $\bar{A}_{2}=V(G) \backslash\left(A_{2} \cup\{s\}\right)$. The analogous statement holds for $A_{3}$.

It follows that $\left|\delta_{G-s}\left(A_{2} \cap A_{3}\right)\right|,\left|\delta_{G-s}\left(A_{2} \backslash A_{3}\right)\right|,\left|\delta_{G-s}\left(A_{3} \backslash A_{2}\right)\right|$, and $\left|\delta_{G-s}\left(\overline{A_{2} \cup A_{3}}\right)\right|$ are all at least $k-1$, while $\left|\delta_{G-s}\left(A_{2}\right)\right|$ and $\left|\delta_{G-s}\left(A_{3}\right)\right|$ are both at most $k-1$. But $k-1$ is odd, so Equation 3.1 cannot be realized (as mentioned in the paragraph following Equation $3.1)$.

We comment that the proofs of Proposition 3.4 and Equation 3.1 also imply that, when $k$ is odd, there is only one pattern for $G$ for which $L(G, s, k)$ is a perfect matching; this is illustrated in Figure 3.5, where there are four edges incident with $s$ and the thick edges represent $(k-1) / 2$ edges. No two edges consecutive in the illustrated cyclic rotation at $s$ form a feasible pair. 


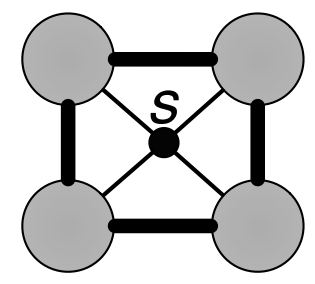

Figure 3.5: Each thick edge represents $(k-1) / 2$ edges.

\section{$3.3 \operatorname{deg}(s)=5$}

In this subsection, we prove the following, dealing with the case $\operatorname{deg}(s)=5$.

Proposition 3.6 Let $k$ be a natural number, and let $G$ be a graph with a vertex $s$ such that any two vertices in $G-s$ are joined by $k$ pairwise edge-disjoint paths in $G$. If $\operatorname{deg}(s)=5$, then $L(G, s, k)$ is either an isolated vertex plus a 4 -cycle or a connected graph. If $k$ is even and $L(G, s, k)$ is connected, then $G$ is a complete multipartite graph.

Proof. Lemma 3.3 (3.3.1) implies the largest independent set in $L(G, s, k)$ has size at most 3 . We break the proof into two cases.

Case 1: $L(G, s, k)$ contains an independent set of size 3.

Let $F$ be an independent set in $L(G, s, k)$ of size 3 and let $A_{1}$ be a dangerous set in $G$ so that the non-s ends of the edges in $F$ are all in $A_{1}$. As there are only two edges incident with $s$ and not in $F$, they both have their non- $s$ ends in $\bar{A}_{1}=V(G) \backslash\left(A_{1} \cup\{s\}\right)$. In particular, $\left|\delta_{G}\left(A_{1}\right)\right|=k+1$ and $\left|\delta_{G}\left(A_{1} \cup\{s\}\right)\right|=k$, so the two edges in $\delta(\{s\}) \backslash F$ are also independent in $L(G, s, k)$.

Suppose $e_{1} \in F$ and $e_{2} \in \delta(\{s\}) \backslash F$ do not form a feasible pair and let $A_{2}$ be a dangerous set that witnesses this. As in the preceding paragraph, there are at least two edges in $\delta(\{s\}) \backslash\left\{e_{1}, e_{2}\right\}$ having their non- $s$ ends in $\bar{A}_{2}$; at least one of these is in $F \backslash\left\{e_{1}\right\}$.

Thus, there is at least one edge from $s$ to each of $A_{1} \cap A_{2}$ (namely, $\left.e_{1}\right), A_{2} \backslash A_{1}\left(e_{2}\right)$, and $A_{1} \backslash A_{2}$ (the one at the end of the preceding paragraph).

If $\overline{A_{1} \cup A_{2} \cup\{s\}} \neq \varnothing$, then Lemma 3.2 implies $3+\left|\delta(\{s\}) \cap \delta\left(A_{2}\right)\right| \leq 4$. But $e_{1}, e_{2} \in$ $\delta(\{s\}) \cap \delta\left(A_{2}\right)$, so we deduce that $\overline{A_{1} \cup A_{2} \cup\{s\}}=\varnothing$.

It follows that both edges in $\delta(\{s\}) \backslash F$ have their non-s ends in $A_{2} \backslash A_{1}$. Thus, $\left|\delta(\{s\}) \cap \delta\left(A_{2}\right)\right| \geq 3$. Since $A_{2}$ is dangerous, Lemma 3.3 implies $\left|\delta(\{s\}) \cap \delta\left(A_{2}\right)\right| \leq 3$. Therefore there are also two edges in $\delta(\{s\})$ with ends in $A_{1} \backslash A_{2}$.

An immediate consequence of the preceding is that $e_{1}$ has no feasible lift with any other edge in $\delta(\{s\})$. Frank's Theorem implies that there is at most one edge incident 
with $s$ that is not in any feasible pair. It follows that $e_{1}$ is the only such edge; now applying the above argument to another edge $e_{1}^{\prime}$ in $F \backslash\left\{e_{1}\right\}$ and an edge $e_{2}$ in $\delta(\{s\}) \backslash F$ shows $e_{1}^{\prime}, e_{2}$ is a feasible pair.

We conclude that, in the event there is an independent set of size 3 in $L(G, s, k)$, $L(G, s, k)$ is either $K_{2,3}$ or an isolated vertex plus $C_{4}$.

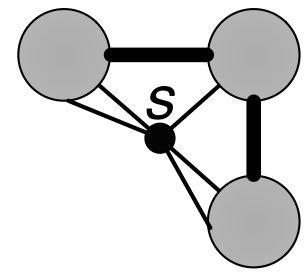

Figure 3.7: If each thick edge represents $k-2$ edges, then $L(G, s, k)$ is an isolated vertex and $C_{4}$. Changing one thick edge to $k-1$ edges turns $L(G, s, k)$ into $K_{2,3}$.

Case 2: every independent set in $L(G, s, k)$ has size at most 2.

Suppose there are three edges $e_{0}, e_{1}, e_{2}$ in $\delta(\{s\})$ such that neither $e_{0}, e_{1}$ nor $e_{0}, e_{2}$ is a feasible pair.

(F1) The assumption of this case implies $e_{1}, e_{2}$ is a feasible pair.

For $i=1,2$, let $A_{i}$ be a dangerous set containing the non-s ends of both $e_{0}$ and $e_{i}$. Because we are in Case 2, none of the three edges in $\delta(\{s\}) \backslash\left\{e_{0}, e_{i}\right\}$ has an end in $A_{i}$. Thus, each of these three edges has an end in $\overline{A_{i} \cup\{s\}}$. Since these three edges do not make an independent set in $L(G, s, k),\left|\delta\left(\overline{A_{i} \cup\{s\}}\right)\right|>k+1$. Evidently, $\left|\delta\left(A_{i}\right)\right| \leq k+1$, so $\left|\delta\left(A_{i}\right)\right|=k+1$.

Moreover, there is precisely one edge from $\delta(\{s\})$ having an end in each of $A_{1} \cap A_{2}$ $\left(e_{0}\right), A_{2} \backslash A_{1}\left(e_{2}\right)$, and $A_{1} \backslash A_{2}\left(e_{1}\right)$. Therefore, the remaining two edges have their non- $s$ ends in $\overline{A_{1} \cup A_{2} \cup\{s\}}$.

Since $\left\{e_{0}, e_{1}, e_{2}\right\}$ is not an independent set of size $3,\left|\delta_{G}\left(A_{1} \cup A_{2}\right)\right| \geq k+2$. Thus, each of $\delta_{G-s}\left(A_{1} \cap A_{2}\right), \delta_{G-s}\left(A_{2} \backslash A_{1}\right), \delta_{G-s}\left(A_{1} \backslash A_{2}\right)$, and $\delta_{G-s}\left(\overline{A_{1} \cup A_{2} \cup\{s\}}\right)$ has size at least $k-1$ (as this is trivially true for the first three). Since $\delta_{G-s}\left(A_{1}\right)$ and $\delta_{G-s}\left(A_{2}\right)$ have size precisely $k-1$, as before from Equation 3.1, $k-1$ is even.

It follows that, for $k$ even, $e_{0}, e_{1}$, and $e_{2}$ do not exist, so $L(G, s, k)$ is complete multipartite.

In the case $k$ is odd, $\left|\delta_{G}\left(\overline{A_{1} \cup A_{2} \cup\{s\}}\right)\right|=k+1$, showing the following.

(F2) The pair $e_{3}, e_{4}$ of edges in $\delta(\{s\}) \backslash\left\{e_{0}, e_{1}, e_{2}\right\}$ is not feasible. 
Subcase 2.1: $e_{1}, e_{3}$ is not feasible.

Applying (F1) to $e_{1}, e_{0}$ and $e_{1}, e_{3}$, we see that $e_{0}, e_{3}$ is a feasible pair.

On the other hand, (F2) implies the pair of edges $e_{2}, e_{4}$ in $\delta(\{s\}) \backslash e_{1}, e_{0}, e_{3}$ is not feasible. Now using $e_{2}, e_{0}$ and $e_{2}, e_{4}$, we conclude from (F1) that $e_{0}, e_{4}$ is feasible.

Finally, (F1) and the infeasible pairs $e_{3}, e_{1}$ and $e_{3}, e_{4}$ show $e_{1}, e_{4}$ is feasible, and analogously $e_{2}, e_{3}$ is feasible. In this case, $L(G, s, k)$ is $C_{5}$.

Subcase 2.2: no version of Subcase 2.1; that is, $\left\{e_{1}, e_{2}, e_{3}, e_{4}\right\}$ induces $K_{4}-e_{3} e_{4}$ in $L(G, s, k)$.

(We remark that this subcase occurs in the version of Figure 3.8 with one thick edge being $(k+1) / 2$ edges.) Suppose $e_{0}, e_{3}$ is not a feasible pair. Then (F2) applied to $e_{0}, e_{1}, e_{3}$ yields the contradiction that $e_{2}, e_{4}$ is not feasible. Therefore, $e_{0}, e_{3}$ and, symmetrically, $e_{0}, e_{4}$, are feasible pairs. In this final case, $L(G, s, k)$ is $K_{5}-\left\{e_{0} e_{1}, e_{0} e_{2}, e_{3} e_{4}\right\}$.

Figure 3.8 gives two examples for odd $k$. One has $L(G, s, k)$ being a 5 -cycle, while, for the other, $L(G, s, k)$ is $K_{5}-\left\{e_{0} e_{1}, e_{0} e_{2}, e_{3} e_{4}\right\}$.

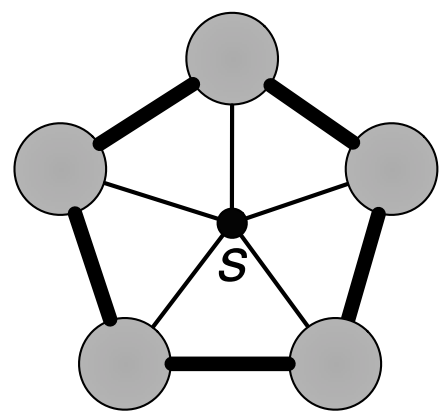

Figure 3.8: If each thick edge represents $(k-1) / 2$ edges, then $L(G, s, k)=C_{5}$. Changing one thick edge to $(k+1) / 2$ edges turns $L(G, s, k)$ into $K_{5}-\left\{e_{0} e_{1}, e_{0} e_{2}, e_{3} e_{4}\right\}$.

\subsection{The inductive step}

In this subsection, we proceed with the induction to complete the proof of Theorem 1.2.

Proof of Theorem 1.2. For (1.2.1), we observe that if $\operatorname{deg}(s)=4$ or 5 , then $L(G, s, k)$ has at most two components. For the induction, suppose $\operatorname{deg}(s) \geq 6$. If $L(G, s, k)$ has more than two components, then it is the union of three subgraphs $J_{1}, J_{2}, J_{3}$, with each $J_{i}$ a union of components of $L(G, s, k)$.

Suppose, for some $i \in\{1,2,3\}, J_{i}$ has at least three vertices. Frank's Theorem implies $J_{i}$ has an edge $e_{1} e_{2}$. Lifting $e_{1} e_{2}$ produces a graph $G^{\prime}$ with $\operatorname{deg}_{G^{\prime}}(s)=\operatorname{deg}_{G}(s)-2$ and 
there is no edge of $L\left(G^{\prime}, s, k\right)$ between any two of the $J_{j} \cap L\left(G^{\prime}, s, k\right)$. This contradicts the inductive assumption that $L\left(G^{\prime}, s, k\right)$ has at most two components.

Therefore, each $J_{i}$ has at most two vertices; since $\operatorname{deg}(s) \geq 6$, each $J_{i}$ has precisely two vertices and $\operatorname{deg}(s)=6$. However, in this case, there are 8 different independent sets of size 3 , each consisting of one vertex from each of the $J_{i}$. This contradicts Lemma 3.3 (3.3.2), completing the proof of (1.2.1).

For (1.2.2), the claim holds for $\operatorname{deg}(s)=5$, so suppose $\operatorname{deg}(s) \geq 7$. Let $H$ and $J$ be the components of $L(G, s, k)$ with $|V(H)|<|V(J)|$. Then $|V(J)| \geq 4$ and if we lift an edge from $J$ to get the graph $G^{\prime}$, there is still no edge between $H \cap L\left(G^{\prime}, s, k\right)$ and $J \cap L\left(G^{\prime}, s, k\right)$ and the latter has at least two vertices. Thus, $H \cap L\left(G^{\prime}, s, k\right)$, and therefore $H$, has only one vertex, as required.

To see that $J$ is complete multipartite, suppose there exist $e_{0}, e_{1}, e_{2}$ in $V(J)$ such that $e_{0}$ is not adjacent in $J$ to either of $e_{1}$ and $e_{2}$, while $e_{1} e_{2} \in E(J)$. Lift the pair $e_{1}, e_{2}$ to get the graph $G^{\prime}$. Since $J$ has at least 6 vertices, $J \cap L\left(G^{\prime}, s, k\right)$ is a component of $L\left(G^{\prime}, s, k\right)$ with at least 4 vertices. By the inductive assumption, it is not a star, so it has an edge $e_{3} e_{4}$ not incident with $e_{0}$. Then $e_{3} e_{4}$ is an edge of $J$.

Lift $e_{3}, e_{4}$ in $G$ to get $G^{\prime \prime}$; the pair $e_{1}, e_{2}$ is feasible in $G^{\prime \prime}$ (the resulting graph is the same as first lifting $e_{1}, e_{2}$ and then lifting $\left.e_{3}, e_{4}\right)$, so $e_{1} e_{2}$ is an edge in $J \cap L\left(G^{\prime \prime}, s, k\right)$. But neither $e_{0} e_{1}$ nor $e_{0} e_{2}$ is an edge in $J \cap L\left(G^{\prime \prime}, s, k\right)$, contradicting the inductive assumption applied to $L\left(G^{\prime \prime}, s, k\right)$. Thus, $J$ is both complete multipartite and not a star, as required.

For (1.2.3), we first prove that every component of $L(G, s, k)$ has an even number of vertices; this is trivial if there is only one component. This is known for $\operatorname{deg}(s)=4$, so we suppose $\operatorname{deg}(s) \geq 6$. Let $H$ and $J$ be the two components with $|V(H)| \leq|V(J)|$. Let $e_{1} e_{2}$ be an edge of $J$ and let $G^{\prime}$ be the result of lifting the pair $e_{1}, e_{2}$. Then $H \cap L\left(G^{\prime}, s, k\right)$ and $J \cap L\left(G^{\prime}, s, k\right)$ are the two components of $L\left(G^{\prime}, s, k\right)$. By induction they each have an even number of vertices, so this also holds for $L(G, s, k)$.

If $\operatorname{deg}(s)=6$, then the induction and Lemma 3.3 (3.3.2) imply that $L(G, s, k)$ is the disjoint union of $K_{2}$ and either $C_{4}$ or $K_{4}$. Therefore, we may assume $\operatorname{deg}(s) \geq 8$.

Case 1: both components of $L(G, s, k)$ have at least four vertices.

Suppose by way of contradiction that there are vertices $e_{0}, e_{1}, e_{2}$ in the component $K$ of $L(G, s, k)$ such that neither $e_{0} e_{1}$ nor $e_{0} e_{2}$ is an edge of $K$, while $e_{1} e_{2}$ is an edge of $K$. Let $J$ be the other component of $L(G, s, k)$.

Lift $e_{1}, e_{2}$ to get $G^{\prime}$. Then $K \cap L\left(G^{\prime}, s, k\right)$ and $J \cap L\left(G^{\prime}, s, k\right)$ are the two components of $L\left(G^{\prime}, s, k\right)$. Thus, there is an edge $e_{3} e_{4}$ in $J \cap L\left(G^{\prime}, s, k\right)$. Now lift $e_{3}, e_{4}$ in $G$ to get $G^{\prime \prime}$. Then $K \cap L\left(G^{\prime \prime}, s, k\right)$ is a component of $L\left(G^{\prime \prime}, s, k\right)$. The edge $e_{1} e_{2}$ is in $K \cap L\left(G^{\prime \prime}, s, k\right)$, while neither $e_{0} e_{1}$ nor $e_{0} e_{2}$ is an edge of $K \cap L\left(G^{\prime \prime}, s, k\right)$. This contradicts the inductive 
assumption that $K \cap L\left(G^{\prime \prime}, s, k\right)$ is complete multipartite.

Case 2: one component of $L(G, s, k)$ has precisely two vertices.

Let $J$ and $K$ be the components of $L(G, s, k)$ so that $J$ has precisely two vertices; thus $K$ has at least six vertices. Suppose $e_{0}, e_{1}, e_{2}$ are vertices of $K$ such that neither $e_{0} e_{1}$ nor $e_{0} e_{2}$ is an edge of $K$, yet $e_{1} e_{2}$ is an edge of $K$.

Lift $e_{1}, e_{2}$ to obtain the graph $G^{\prime}$. By the induction, $K \cap L\left(G^{\prime}, s, k\right)$ is a component of $L\left(G^{\prime}, s, k\right)$, and it has at least 4 vertices, so it is not a star. Therefore, it has an edge $e_{3} e_{4}$ disjoint from $e_{0}$; we lift $e_{3}, e_{4}$ in $G$ to obtain $G^{\prime \prime}$. Induction tells us that $K \cap L\left(G^{\prime \prime}, s, k\right)$ is complete multipartite, which contradicts the fact that $e_{0}, e_{1}, e_{2}$ are all in $K \cap L\left(G^{\prime \prime}, s, k\right)$, $e_{0} e_{1}$ and $e_{0} e_{2}$ are not edges, and $e_{1} e_{2}$ is an edge.

Lastly, we prove (1.2.4). Proposition 3.4 gives the result for $\operatorname{deg}(s)=4$, so we assume $\operatorname{deg}(s) \geq 6$. Suppose $e_{0}, e_{1}, e_{2}$ are vertices in $L(G, s, k)$ such that $e_{0}$ is not adjacent to either $e_{1}$ or $e_{2}$, but $e_{1} e_{2}$ is an edge of $L(G, s, k)$. Lifting $e_{1}, e_{2}$ yields a graph $G^{\prime}$ for which $L\left(G^{\prime}, s, k\right)$ has at least 4 vertices. By induction, $L\left(G^{\prime}, s, k\right)$ is connected, complete multipartite, and not a star; in particular, it has an edge $e_{3} e_{4}$ disjoint from $e_{0}$.

Lifting $e_{3}, e_{4}$ in $G$ produces a graph $G^{\prime \prime}$; by induction $L\left(G^{\prime \prime}, s, k\right)$ is complete multipartite. However, $e_{0}$ is still not adjacent to either $e_{1}$ or $e_{2}$, while $e_{1} e_{2}$ is an edge. This contradiction shows $L(G, s, k)$ is complete multipartite and Frank's Theorem [3] shows it is not a star, as required.

We conclude this section with Figure 3.9. This is an example having $\operatorname{deg}(s)=6$ and $k=5$ so that $L(G, s, k)$ is $K_{3,3}$ minus an edge; in particular, it is connected and not complete multipartite. The three edges incident with $s$ on the left side are one independent set, the three on the right are a second, and the two going to the bottom are not feasible.

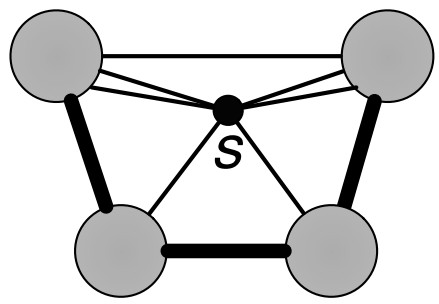

Figure 3.9: Each thick edge represents 2 edges and $k=5$. 


\section{Weakly $k$-linked infinite graphs}

In this section we prove Theorem 1.3: if $k$ is odd, then a $(k+2)$-edge-connected, locally finite, 1-ended, infinite graph $G$ is weakly $k$-linked.

If $\mathbf{x}=\left(x_{1}, x_{2}, \ldots, x_{k}\right)$ and $\mathbf{y}=\left(y_{1}, y_{2}, \ldots, y_{k}\right)$ are sequences of (not necessarily distinct) vertices in graph $G$, then an xy-linkage is a set $\left\{P_{1}, P_{2}, \ldots, P_{k}\right\}$ of pairwise edgedisjoint paths in $G$ such that, for $i=1,2, \ldots, k, P_{i}$ is an $x_{i} y_{i}$-path.

Before we prove Theorem 1.3, we require extensions of the theorems of Mader and Frank and of our Theorem 1.2 to locally finite graphs. These extensions may all be proved as follows. Let $G_{d}$ be the subgraph of a locally finite graph $G$ consisting of those vertices at distance at most $d$ from the specified vertex $s$. Let $G_{d}^{\prime}$ be the graph obtained from $G$ by contracting each component of $G-V\left(G_{d}\right)$ to a vertex. For infinitely many $d$, the lifting graph $L\left(G_{d}^{\prime}, s, \tau\right)$ is the same graph; this is the the lifting graph $L(G, s, \tau)$.

Proof of Theorem 1.3. Let $\mathbf{x}$ and $\mathbf{y}$ be any sequences of $k$ (not necessarily distinct) vertices of $G$. Let $A$ be the set of vertices that occur in $\mathbf{x}$ and $\mathbf{y}$.

Let $S$ be a finite set of vertices containing $A$. There is a unique infinite component $K$ of $G-S$. Let $\mathcal{P}$ be a largest set of pairwise edge-disjoint, 1-way infinite paths (or rays), that begin with an edge in $\delta(V(K))$ and are otherwise contained in $K$. It is a standard fact that there is a finite set $S^{\prime}$ containing $S$ such that $\left|\delta\left(S^{\prime}\right)\right|=|\mathcal{P}|$. We are interested only in $S^{\prime}$, which we relabel as $S$, and restrict the rays in $\mathcal{P}$ to begin at their edge in $\delta\left(S^{\prime}\right)$.

Because $G$ is $(k+2)$-edge-connected, $|\delta(S)| \geq k+2$. We consider three cases.

Case 1: $|\delta(S)|=k+2$.

Contract $G-S$ to a single vertex $v_{S}$, yielding a finite $(k+2)$-edge-connected graph $G /(G-S)$. Huck's Theorem shows there is a weak xy-linkage $\mathcal{L}$ in $G /(G-S)$.

Let $v$ be any vertex of $G-S$. There is a set $\mathcal{L}^{\prime}$ of $(k+2)$ pairwise edge-disjoint paths with origin $v$ whose other end is in $S$ and incident with an edge of $\delta(S)$. Evidently, we can replace any passage of a path in $\mathcal{L}$ through $v_{S}$ with an appropriate pair of paths in $\mathcal{L}^{\prime}$. Simplifying the resulting walks as needed, we convert $\mathcal{L}$ into a weak xy-linkage in $G$.

Case 2: $|\delta(S)|$ is odd and at least $k+4$.

In this case, let $e$ be any edge of $\delta(S)$ and let $G^{\prime}=G-e$. Now $G^{\prime}$ is $(k+1)$-edgeconnected and $|\delta(S)|$ is even. We now proceed as in Case 3.

Case 3: $|\delta(S)|$ is even.

In this case, we need only that $G$ is $(k+1)$-edge-connected (so Case 2 continues smoothly here). Contract $G-S$ to a single vertex $v_{S}$ resulting in the finite graph $G^{S}$. 
We claim that $\delta(S)$ partitions into $|\delta(S)| / 2$ pairs $\left\{e_{i}, e_{i}^{\prime}\right\}, i=1,2, \ldots,|\delta(S)| / 2$, such that, letting $G_{0}^{S}=G^{S}$ and, for $i=1,2, \ldots,|\delta(S)| / 2, G_{i}^{S}$ is the graph obtained from lifting $\left\{e_{i}, e_{i}^{\prime}\right\}$ in $G_{i-1}^{S}$ :

1. for $i \geq 1$, the pair $\left\{e_{i}, e_{i}^{\prime}\right\}$ is $(k+1)$-liftable in $G_{i-1}^{S}$; and

2. for $i=1,2, \ldots,|\delta(S)| / 2$, there is a path $P_{i}$ joining $e_{i}$ and $e_{i}^{\prime}$ with only its end vertices and $e_{i}, e_{i}^{\prime}$ not in $G-S$ such that $P_{i}$ is edge-disjoint from $P_{1} \cup \cdots \cup P_{i-1}$ and from all the rays in $\mathcal{P}$ containing $e_{i+1}, e_{i+1}^{\prime}, \ldots, e_{|\delta(S)| / 2}, e_{|\delta(S)| / 2}^{\prime}$.

Suppose we have the pairs $\left\{e_{1}, e_{1}^{\prime}\right\}, \ldots,\left\{e_{i-1}, e_{i-1}^{\prime}\right\}$ and paths $P_{1}, \ldots, P_{i-1}$. We show the existence of $\left\{e_{i}, e_{i}^{\prime}\right\}$ and $P_{i}$.

Set $\delta_{i}(S)$ to be $\delta(S) \backslash\left\{e_{1}, e_{1}^{\prime}, \ldots, e_{i-1}, e_{i-1}^{\prime}\right\}$. These are the edges in $G-\left\{e_{1}, e_{1}^{\prime}, \ldots, e_{i-1}\right.$, $\left.e_{i-1}^{\prime}\right\}$ having precisely one end in $S$. Let $\mathcal{P}_{i}$ denote the paths in $\mathcal{P}$ that do not contain any of the edges in $\left\{e_{1}, e_{1}^{\prime}, \ldots, e_{i-1}, e_{i-1}^{\prime}\right\}$.

There are two graphs with vertex set $\delta_{i}(S)$ that are relevant to completing the proof.

In the end graph $\mathcal{E}_{i}$, distinct edges $e, e^{\prime}$ in $\delta_{i}(S)$ are adjacent if there are infinitely many vertex-disjoint paths in $G-S$ that: (i) join the two paths in $\mathcal{P}_{i}$ containing $e$ and $e^{\prime}$; and (ii) are edge-disjoint from all the other paths in $\mathcal{P}_{i}$. Since all the paths in $\mathcal{P}_{i}$ are in the same end, $\mathcal{E}_{i}$ is connected.

The other graph is the $(k+1)$-lifting graph $\mathcal{L}_{i}$ for $v_{S}$ in $G_{i-1}^{S}$. By Theorem 1.2 (1.2.4), $\mathcal{L}_{i}$ is a complete multipartite graph. Therefore, its complement is disconnected.

Since $\mathcal{E}_{i}$ is connected, there is an edge $e_{i} e_{i}^{\prime}$ of $\mathcal{E}_{i}$ that is not in the complement of $\mathcal{L}_{i}$; that is, $e_{i} e_{i}^{\prime}$ is an edge of $\mathcal{L}_{i}$. This is the required next pair of edges.

Let $Q$ and $Q^{\prime}$ be the rays in $\mathcal{P}$ containing $e_{i}$ and $e_{i}^{\prime}$, respectively. Because $e_{i} e_{i}^{\prime}$ is an edge of $\mathcal{E}_{i}$, there are infinitely many vertex-disjoint paths in $G$ joining $Q$ and $Q^{\prime}$ that are edge-disjoint from the other rays in $\mathcal{P}_{i}$. Let $P$ be one of these contained in $G-S$ that is also disjoint from all of the finitely many finite paths $P_{1}, \ldots, P_{i-1}$. Then $Q \cup P \cup Q^{\prime}$ contains a path $P_{i}$ containing $e_{i}$, and $e_{i}^{\prime}$. This is the required next path.

The choices of the lifts $\left\{e_{i}, e_{i}^{\prime}\right\}$ show that $G_{|\delta(S)| / 2}^{S}$ is $(k+1)$-connected. Huck's Theorem shows that $G_{|\delta(S)| / 2}^{S}$ has an xy-linkage $\mathcal{Q}$.

An occurrence of the lift of $\left\{e_{i}, e_{i}^{\prime}\right\}$ in some path in $\mathcal{Q}$ can be replaced by $P_{i}$. This converts $\mathcal{Q}$ into an xy-linkage in $G$, as required.

\section{References}

[1] R. Aharoni and C. Thomassen, Infinite, highly connected digraphs with no two arcdisjoint spanning trees. J. Graph Theory 13 (1989), 71-74. 
[2] Y.H. Chan, W.S. Fung, L.C. Lau, and C.K Yung, Degree bounded network design with metric costs, SIAM J. Comput. 40 (2011), no. 4, 953-980.

[3] A. Frank, On a theorem of Mader, Ann. of Disc. Math. 101 (1992), 49-57.

[4] A. Huck, A sufficient condition for graphs to be weakly $k$-linked, Graphs and Combinatorics 7 (1991), 323-351.

[5] W. Mader, A reduction method for edge-connectivity in graphs, Ann. of Disc. Math. 3 (1978), 145-164.

[6] H. Okamura, Every $4 k$-Edge-Connected Graph is weakly-3k-linked. Graphs and Combinatorics 6 (1990), 179185.

[7] C. Thomassen, 2-linked graphs, Europ. J. Comb. 1 (1980), 371-378.

[8] C. Thomassen, Orientations of infinite graphs with prescribed edge-connectivity, Combinatorica, to appear. 